\title{
Distribución potencial y conectividad del paisaje: criterios para reevaluar el grado de amenaza de Campylorhynchus yucatanicus (Aves: Troglodytidae)
}

\author{
Anay Serrano-Rodríguez ${ }^{1}$, Griselda Escalona-Segura ${ }^{1}$, Alexis H. Plasencia Vázquez ${ }^{2}$, \\ Eduardo E. Iñigo Elias ${ }^{3} \&$ Lorena Ruiz-Montoya ${ }^{1}$ \\ 1. El Colegio de la Frontera Sur. Avenida Rancho Polígono 2-A Ciudad Industrial, Lerma, Campeche, Campeche, \\ México CP. 24500; anayserrano1984@gmail.com, gescalon@ecosur.mx, 1ruiz@ecosur.mx \\ 2. Universidad Autónoma de Campeche. Avenida Agustín Melgar, Colonia Buenavista, Campeche, Campeche, México \\ CP 24039; alexpla79@gmail.com \\ 3. Cornell Lab of Ornithology. 159 Sapsucker Woods Rd Ithaca, NY 14850, USA Ithaca, NY, EUA; eei2@cornell.edu
}

Recibido 06-III-2017. Corregido 07-VII-2017. Aceptado 08-VIII-2017.

\begin{abstract}
Potential distribution and landscape connectivity: criteria for reevaluating the threat degree of Campylorhynchus yucatanicus (Aves: Troglodytidae). Geographic distribution and habitat quality are key criteria for assessing the degree of risk of species extinction threat. Campylorhynchus yucatanicus (Yucatán Wren, Troglodytidae) is an endemic bird of the Northern coast of the Yucatán Peninsula, Mexico, with a distribution restricted to a narrow strip of habitat, between Campeche and Yucatán states. Currently, the Yucatán coast has lost more than half of the coastal dune vegetation, and other habitats have been modified mainly because there is no urban development plan and the natural resources management is poor. These factors threaten C. yucatanicus, which is listed as a near threatened species by IUCN and as an endangered species by Mexican law NOM059-2010. In this paper, C. yucatanicus's potential distribution was modeled using 64 presence records from several sources (1960 y 2009), a set of climate variables, and a vegetation index layer of normalized difference (NDVI). To assess the degree of landscape connectivity we used a map of vegetation types and land use, distance to villages and paved roads. The potential distribution model showed an area of approximately $2711 \mathrm{~km}^{2}$, which is $2 \%$ of the total area of the Yucatán Peninsula distribution. In this area, only $27 \%$ is protected by Biosphere Reserve category and only $10 \%$ belong to core conservation areas, with land use restrictions and relatively effective protection. The populations from Ría Lagartos and Western Celestún regions appear to be the most isolated following the model of landscape connectivity. Landscape permeability among fragments of dune vegetation near the coast is low, mainly due to the distribution of urban areas. These results can be used to establish management strategies, and show that the species is in more delicate conditions than what it has been described by IUCN. We consider that $C$. yucatanicus should be given endangered category by IUCN, because of their distribution and the context of the current landscape connectivity. Rev. Biol. Trop. 65 (4): 1554-1568. Epub 2017 December 01.
\end{abstract}

Key words: endemic, threatened, Yucatan Wren, potential distribution, landscape connectivity.

La fragmentación antropogénica del hábitat es considerada una de las mayores amenazas a la biodiversidad en el mundo (Naranjo et al., 2009; Keith, 2015). Como una consecuencia directa, ha disminuido de forma abrupta la conectividad entre las poblaciones de varias especies de aves, lo cual puede provocar la extinción de poblaciones aisladas (Bellard,
Bertelsmeier, Leadley, Thuiller, \& Courchamp, 2012; Modest \& Hassan, 2016). A este fenómeno son generalmente más vulnerables las especies de distribución restringida o aquellas que son hábitat específicas (Dyer et al., 2016). Surgen entonces los estudios que involucran modelos de distribución potencial de especies (SDM) y análisis espaciales, como por ejemplo 
la conectividad del paisaje, como una herramienta importante no solo para investigaciones teóricas en ecología y procesos evolutivos, sino para elaborar estrategias de conservación de estas especies en particular y de la biodiversidad en general (Guisan et al., 2006; Kozak, Graham, \& Wiens, 2008; Correa Ayram, Mendoza, Etter, \& Pérez Salicrup, 2016). En ese sentido se dirigen una gran cantidad de métodos cada vez más eficientes que buscan obtener patrones espaciales con los datos disponibles hasta el momento (McRae, Dickson, Keitt, \& Shah, 2008; Garrido-Garduño \& VázquezDomínguez, 2013).

La respuesta de los organismos a la estructura del paisaje depende de muchos factores entre los que se encuentran la escala en la que perciban la heterogeneidad del mismo y la habilidad que tengan para moverse (Tischendorf \& Fahrig, 2000). Entre las aproximaciones más ampliamente difundidas para analizar la conectividad del paisaje, están aquellas basadas en modelos de circuitos (McRae et al., 2008), donde se puede caracterizar el paisaje espacialmente de una manera explícita. Esto permite evaluar la importancia de los elementos individuales para el mantenimiento o fomento de la conectividad del paisaje en su conjunto, constituyendo una herramienta muy útil para orientar decisiones dirigidas al manejo de vida silvestre (Koen, Garroway, Wilson, \& Bowman, 2010; Shimazaki et al., 2016).

Diseñar planes ambientales adecuados es un gran reto, porque no se cuenta con la información básica necesaria que permita, en primer lugar, definir con certeza especies y áreas prioritarias para la conservación (Stotz, Fitzpatrick, Parker, \& Moskovits, 1996; Alaniz, Galleguillos, \& Perez-Quezada, 2016). En México, se han establecido una gran cantidad de reservas y áreas naturales protegidas (ANP) con el objetivo de salvaguardar ecosistemas, especies, y biodiversidad en general; sin embargo, en muchas de ellas no existe infraestructura ni información para poder llevar a cabo un plan de manejo adecuado (Bezaury-Creel \& Gutiérrez-Carbonell, 2009; Maass et al., 2010). Se necesitan estrategias de conservación que incluyan especies correctamente catalogadas por su riesgo de amenaza e identificar áreas de hábitats potencialmente adecuados dentro y fuera de las ANP actuales, que actúen como corredores y permitan una conexión entre las poblaciones de interés (Rödder, Nekum, Cord, \& Engler, 2016).

En la actualidad, se estima que la costa de la Península de Yucatán ha perdido más de la mitad de la extensión territorial del complejo de vegetación de duna costera, principalmente por obras de urbanización y por el mal aprovechamiento que se ha hecho de los recursos naturales (Durán, Torres, \& Espejel, 2010). A medida que el matorral costero y la vegetación aledaña se han perdido, la distribución de las especies asociadas también podría afectarse. De las aves registradas en la Península, Campylorhynchus yucatanicus (Matraca Yucateca, Troglodytidae) es una de las que presenta distribución más restringida (Paynter, 1955; Ceballos, Gómez Silva, \& del Coro Arizmendi, 2002; Navarro-Sigüenza \& Peterson, 2007, Vázquez-Miranda, Navarro-Sigüenza, \& Omland, 2009; MacKinnon, 2013; Berlanga et al., 2015). Esta ave es endémica y su ocurrencia se limita a una estrecha franja costera del norte de la Península, entre los estados de Campeche y Yucatán (Howell \& Webb, 1995; Del Hoyo, Elliot, \& Christie, 2005; MacKinnon, 2013). Se ha descrito que habita en el matorral costero espinoso (Zimmerman, 1957), el cual es considerado como raro y de poca extensión (Espejel, 1984; Flores \& Espejel, 1994; 1987), aunque ocasionalmente se registran individuos en el borde de manglares y malezas de sitios parcialmente perturbados cercanos a la costa (del Hoyo et al., 2005). Las principales amenazas de C. yucatanicus que se reconocen son el desarrollo de infraestructura hotelera, expansión de comunidades rurales y en menor medida la recolecta de leña y la tala (CONANP, 2000; Vargas-Soriano, Salgado-Ortíz, \& Escalona-Segura, 2010). Es por eso que la especie está catalogada como en peligro de extinción por la NOM-059-2010 (SEMARNAT, 2010); sin embargo, la IUCN (2015) la ubica en la categoría de vulnerable. El objetivo de este 
estudio fue determinar el área de distribución geográfica potencial de C. yucatanicus y la representatividad de esta en el sistema nacional de áreas protegidas, así como evaluar la conectividad del paisaje con vistas a reconsiderar la categoría de amenaza de la especie.

\section{MATERIALES Y MÉTODOS}

Área de estudio: El presente estudio se llevó a cabo en la costa norte de la Península de Yucatán, que incluye los estados mexicanos de Campeche, Quintana Roo y Yucatán. De acuerdo a la clasificación de Köppen modificada por García (2004), la Península posee un clima cálido-húmedo, con un régimen de lluvias en verano, temperatura promedio de $25.7^{\circ} \mathrm{C}$, con mínimos de $18{ }^{\circ} \mathrm{C}$ y máximos de $45^{\circ} \mathrm{C}$. Cuenta con tres épocas climáticas a lo largo del año: "Secas" de febrero a mayo, "Lluvias" de junio a septiembre y "Nortes" de octubre a marzo (García, 2004).

En general, la Península de Yucatán presenta suelos básicamente calcáreos, sin grandes accidentes geográficos y de formación reciente. La mayor parte de este territorio está cubierto por selvas tropicales, como selva baja caducifolia, mediana subcaducifolia, y mediana subperennifolia. Otros tipos de vegetación ocupan un área más reducida en la Península; sin embargo, fueron de mayor interés para este estudio ya que en ellos habita C. yucatanicus. Estos tipos de vegetación fueron la vegetación de dunas costeras, la vegetación arbustiva de manglar, la selva baja espinosa caducifolia y la vegetación secundaria arbórea de selva baja espinosa caducifolia (INEGI, 2014).

En la costa norte de la Península de la Yucatán, están establecidas dos Reservas de la Biosfera (RB) que se ubican en los extremos de la distribución de C. yucatanicus: RB Ría Celestún y RB Ría Lagartos. Adicionalmente, existen tres reservas estatales (RE): RE El Palmar, RE Humedales y manglares de la costa norte de Yucatán y RE Dzilam. Estas RE cubren casi la totalidad de la costa norte de Yucatán comprendida entre las dos RB anteriormente mencionadas.
Modelación de la distribución potencial: Se emplearon registros de presencia de C. yucatanicus, disponibles en las bases de datos del Gbif (http://www.gbif.org) y eBird (http://ebird.org). Entre el total de registros disponibles se eliminaron aquellos que estaban repetidos o muy cercanos entre sí (a menos de $1 \mathrm{~km}$ ), así como aquellos que eran de procedencia dudosa o los que aparecían en áreas donde se conoce que la especie no está presente por trabajo de campo y experiencia de especialistas y observadores de aves. Finalmente, se seleccionaron 64 registros de presencia que cumplieron con los requisitos antes mencionados, y que correspondieron al periodo entre 1960 y 2009.

Para modelar la distribución potencial y el nicho ecológico de C. yucatanicus se analizaron 19 capas bioclimáticas con una resolución espacial de 30' (aproximadamente $1 \mathrm{~km}$ ) derivadas de valores mensuales de precipitación y temperatura (Cuervo et al., 2013). Estas capas de clima describen un periodo comprendido entre 1910 y 2009. Se realizó un análisis de correlación entre pares de variables para desechar aquellas que tenían índices de correlación de Pearson mayores de 0.7 usando el ENMTools (Warren, Glor, \& Turelli, 2008; 2010). Finalmente, se incluyeron nueve variables bioclimáticas en el análisis (Cuadro 1). El criterio para la selección de las variables fue el conocimiento de la biología de la especie, además de los coeficientes de correlación de Pearson antes mencionados.

Adicionalmente, se empleó una capa de índice topográfico de 30' de resolución (http:// gcmd.nasa.gov/records/GCMD_HYDRO1k. html) y dos capas de Índice de Vegetación de Diferencia Normalizada (NDVI) como medida indirecta de la estructura de la vegetación; una de ellas correspondiente al promedio de NDVI en el mes de abril durante cuatro años (del 2006 al 2009, mes seco), y otra del promedio de los mismos años en el mes de octubre (mes húmedo), obtenidos a partir del satélite MODIS (http://earthexplorer.usgs.gov) con una resolución de $250 \mathrm{~m}$. Estas capas fueron remuestreadas a una resolución de 30'. 
CUADRO 1

Variables utilizadas para modelar la distribución potencial y el nicho ecológico potencial de Campylorhynchus yucatanicus en la Península de Yucatán

TABLE 1

Variables used to model the potential distribution and potential ecological niche of Campylorhynchus yucatanicus in the Yucatán Peninsula

\begin{tabular}{lccc}
\multicolumn{1}{c}{ Variable } & Valores propios (PCA) & Vectores propios (PCA) \\
Temperatura media anual (bio1) & 179.5 & -31.4 & -347.6 \\
Intervalo diurno de temperatura (bio2) & 279.1 & -16.3 & -233.2 \\
Desviación estándar de la temperatura (bio4) & 168.5 & -185.2 & -229.9 \\
Máxima temperatura del mes más cálido (bio5) & 593.5 & 8.6 & 13.1 \\
Mínima temperatura del mes más frío (bio6) & 463.4 & -335.6 & -115.4 \\
Precipitación media anual (bio12) & 64.0 & -334.7 & 190.4 \\
Precipitación del mes más seco (bio13) & 85.0 & -295.0 & -157.4 \\
Precipitación del mes más húmedo (bio14) & 2.7 & -386.3 & -77.2 \\
Coeficiente de variación de la precipitación (bio15) & 143.6 & 246.2 & -293.1 \\
Índice topográfico & 6.9 & -21.5 & 197.1 \\
NDVI abril & 14.5 & -170.5 & 143.2 \\
NDVI octubre & 39.1 & 81.4 & -97.3 \\
\hline
\end{tabular}

Se muestran los valores y vectores propios de cada variable que se obtuvieron en el PCA para graficar en dos dimensiones el espacio ambiental con los dos primeros componentes.

Show the values and vectors of each variable obtained in the PCA to graph the environmental space with the first two components in two dimensions.

Los mapas de distribución potencial fueron generados en el programa MaxEnt (Phillips, Anderson, \& Schapire, 2006) y se usó el algoritmo de máxima entropía, que es uno de los de mejor desempeño para conjunto de datos de sólo presencia (Elith \& Graham, 2006). Se obtuvieron 15 modelos y para cada uno se realizaron 1000 iteraciones, en los cuales se empleó el método de remuestreo boopstrap. El $80 \%$ de los datos se utilizaron para obtener los modelos y el resto para la validación de los mismos. La validación del modelo se efectuó a través de los valores de AUC que arroja el MaxEnt y a través del programa ROC parcial (Barve, 2008), como sugieren Peterson, Papes \& Soberon (2008), para mitigar las limitaciones de este método. En este proceso se buscaron las relaciones entre los AUC de las curvas ROC observadas y los valores esperados al azar, empleando un umbral de error esperado de $5 \%$ y 1000 iteraciones. Los valores resultantes se encuentran entre 0 y 2 , donde 1 equivale a un modelo obtenido totalmente por azar y se consideró un buen modelo aquel en el que se obtuvieron valores $>1$.

Para obtener un mapa binario de presencia/ ausencia se aplicaron diferentes líneas de corte a la mediana de los 15 modelos obtenidos. Estas se establecieron teniendo en cuenta cuatro de los algoritmos más utilizados y recomendados en la literatura: el de presencia mínima de los puntos de entrenamiento (minimum training presence, MTP), el 10 percentil (10 percentile training presence, $10 \mathrm{P}$ ), la sensibilidad y especificidad equivalentes (equal training sensitivity and specificity, ETSS) y la de sensibilidad máxima de entrenamiento más especificidad (Maximum training sensitivity plus specificity, MTSPS) (Liu, Berry, Dawson, \& Pearson, 2005; Peterson et al., 2008). Para seleccionar el mejor entre los cuatro algoritmos, se tuvieron en cuenta aspectos descritos de la historia natural de la especie (Zimmerman, 1957; Rabenold, 1990; Howell \& Webb, 1995; Barker, 2007; Vargas-Soriano et al., 2010; MacKinnon, 2013), así como información que se conoce 
por experiencia de campo durante muestreos realizados en áreas donde se localiza C. yucatanicus, ya que el error de omisión no fue diferente entre ellos.

La contribución de las variables ambientales a la predicción del modelo se evaluó analizando los resultados del Jackknife, disponible en el MaxEnt. Se utilizaron además, los valores de la mediana para los porcentajes de contribución relativa de cada variable para los 15 modelos. Para poder visualizar cómo es que las variables ambientales con mayor aporte influyen sobre la predicción del modelo se utilizaron las curvas de respuesta. Estas curvas muestran cómo afecta cada variable ambiental a la predicción de MaxEnt, teniendo en cuenta la probabilidad predicha de condiciones idóneas frente a los valores de cada variable ambiental. (Phillips et al., 2006).

Para analizar la ubicación y tamaño del nicho con respecto a las condiciones ambientales disponibles en el espacio ambiental, se empleó un análisis de componentes principales con las 12 variables utilizadas (Cuadro 1) y graficamos los primeros dos vectores. Los valores máximos y mínimos en el gráfico estuvieron determinados por los valores extremos de las variables ambientales, los cuales se extrajeron de todo el ambiente disponible en el área de estudio en la Península de Yucatán. Para dicho análisis se diseñó un gráfico de $100 \times 100$ cuadrantes, en la que cada celda corresponde a un vector único con una combinación de condiciones ambientales disponibles (Broennimann et al., 2012). En el gráfico se representó la probabilidad de ocurrencia de la especie a través de la densidad de presencia en cada celda del espacio ambiental. Estos valores se estimaron y suavizaron con una función de densidad de Kernel (Silverman, 1986; Broennimann et al., 2012). El análisis se ejecutó en el paquete estadístico R (R Development Core Team 2014) y permitió caracterizar el nicho ecológico potencial de la especie de forma integral a través de la representación gráfica en el espacio ambiental.
Conectividad del paisaje: Para evaluar la resistencia que ofrece el paisaje al movimiento de individuos se utilizó la teoría de circuitos (McRae et al., 2008) implementada en el Circuitscape (McRae \& Shah, 2009). El término resistencia se usó como antónimo de la conectividad del paisaje, que definimos como el grado en el cual el paisaje facilita o impide el movimiento de los individuos (Taylor, Fahrig, Henein, \& Merriam, 1993) que pueden moverse al azar entre dos sitios o nodos. En este contexto, los nodos según la teoría de circuitos en los modelos de conectividad pueden ser parches de hábitat, poblaciones o puntos en el paisaje, entre los cuales nos interesa evaluar conectividad (McRae et al., 2008). Para establecer los nodos focales se utilizaron los puntos de ocurrencia de las bases de datos del Gbif y eBird, además de registros de campo nuestros y de observadores de aves obtenidos entre el 2010 y 2016. Después de depurar los datos de campo quedaron 28 nodos focales, que se corresponden a un conjunto de ocurrencias cercanas entre sí $(<500 \mathrm{~m})$. El área analizada es un recorte del mapa de la Península de Yucatán que contenía la distribución potencial obtenida, lo cual permitió adquirir modelos de resistencia más certeros (Koen et al., 2010). En primera instancia, se confeccionó un mapa de idoneidad de $200 \mathrm{~m}$ de resolución, teniendo en cuenta los tipos de vegetación y usos de suelo (INEGI, 2013), la distancia a los asentamientos humanos y la distancia a las carreteras asfaltadas. A cada una de estas variables se le asignó un peso considerando la relación que tienen sobre la probabilidad de presencia de la especie: 75 al tipo de vegetación, 15 a la distancia a los poblados y 10 a la distancia a las carreteras asfaltadas. El valor de peso de estas variables se estableció a priori teniendo en cuenta nuestras observaciones de campo, y de otros observadores de aves, y la información disponible en la literatura acerca de la ecología de la especie (Paynter, 1955; Zimmerman, 1957; del Hoyo et al., 2005; Vargas-Soriano et al., 2010). De igual manera, se establecieron clases dentro de cada variable, a las cuales le asignamos un valor de importancia para la presencia 
de la especie, desde cero si considerábamos que no era probable que los individuos pudieran hallarse, hasta 100 en aquellas con alta probabilidad de que pudieran estar. Los tipos de vegetación con valores de peso más altos se eligieron de acuerdo a la cantidad de registros de la especie en cada tipo y fueron: vegetación de dunas costeras (100), vegetación arbustiva de manglar y vegetación secundaria arbustiva de selva baja espinosa caducifolia (35), y vegetación secundaria arbórea de selva baja espinosa caducifolia (25).

\section{RESULTADOS}

Modelación de la distribución potencial: El modelo de distribución potencial de C. yucatanicus seleccionado obtuvo un buen desempeño con un valor de 0.99 tanto para los datos de entrenamiento como para los de validación. Los mapas binarios que se lograron usando las líneas de corte con los algoritmos MTP, ETSS y MTSPS fueron semejantes y tendieron a sobreestimar la distribución potencial de la especie, atendiendo a los criterios de la historia natural de C. yucatanicus que se tuvieron en cuenta y los registros de campo actuales obtenidos por nosotros entre 2014 y 2016. Se eligió el algoritmo 10P para confeccionar el mapa binario porque fue el que menos sobreestimó el área de distribución potencial, para lo cual se tuvieron en cuenta los criterios antes mencionados (Fig. 1A y Fig. 1C). Mediante el análisis de la curva ROC parcial se obtuvo un promedio de $1.34 \pm$ 0.12 , en un intervalo entre 1.12 y 1.79 . Estos valores fueron superiores al umbral $(\geq 1)$, lo que indica que el modelo albergó una buena predicción del hábitat adecuado para la presencia de la especie (Peterson et al., 2008), teniendo en cuenta las variables empleadas.

El nicho ecológico de C. yucatanicus estimado en el espacio ambiental no cubrió la totalidad del nicho disponible en el área estudiada (Fig. 1B) y representó menos del $50 \%$ del mismo. Este fue relativamente marginal respecto al ambiente disponible, y sus centros no coinciden. Se observaron dos espacios ambientales separados que describieron el nicho de la especie, uno en el que la densidad de ocurrencias es mayor y otro más pequeño y con densidad de ocurrencias menor. Los dos primeros componentes del PCA explicaron un $61 \%$ de la varianza en el espacio ambiental disponible. Las variables que más aportaron a la varianza del conjunto de datos en todo el espacio ambiental fueron bio5, bio6, bio2 y bio1 en ese orden (Cuadro 1), relacionadas con los factores climáticos de temperatura y precipitación.

Las variables con los mayores porcentajes de aporte al modelo coincidieron con las de mayor contribución según Jackknife. Según este análisis la variable que más aportó fue la precipitación media anual (bio12), con un porcentaje de contribución del $49 \%$. Le siguieron en importancia la precipitación del mes más seco (bio13) con el $31 \%$, y el NDVI de octubre con un $14 \%$ de aporte al modelo.

La distribución potencial de C. yucatanicus estuvo condicionada por variables de precipitación y por la estructura de la vegetación (evaluado a través del NDVI). Según el modelo seleccionado, C. yucatanicus se distribuyó potencialmente en áreas donde las precipitaciones anuales se mantienen sobre los $600 \mathrm{~mm}$; por debajo de este valor fue nula la predicción de ocurrencias (Fig. 2). Por encima de los $600 \mathrm{~mm}$ de precipitación anual la idoneidad del hábitat decreció exponencialmente y a partir de los $900 \mathrm{~mm}$ ya no se predice ninguna presencia. Además, se predijo mayor idoneidad de hábitat en zonas en las cuales durante el mes más lluvioso las precipitaciones son escasas y se mantienen en un intervalo entre los $30 \mathrm{y}$ $80 \mathrm{~mm}$. En relación al NDVI, los resultados indicaron que los hábitats adecuados para $C$. yucatanicus se localizan con mayor frecuencia en áreas con vegetación escasa, con zonas de suelo desnudo que pueden ser rocosos. La idoneidad del hábitat para la presencia de la especie disminuye a medida que la vegetación se hace más densa (Fig. 2).

El área de distribución potencial estimada para C. yucatanicus fue de $2711 \mathrm{~km}^{2}$, lo cual representa aproximadamente un $2 \%$ del área total que ocupa la Península de Yucatán. El mayor porcentaje de área de 


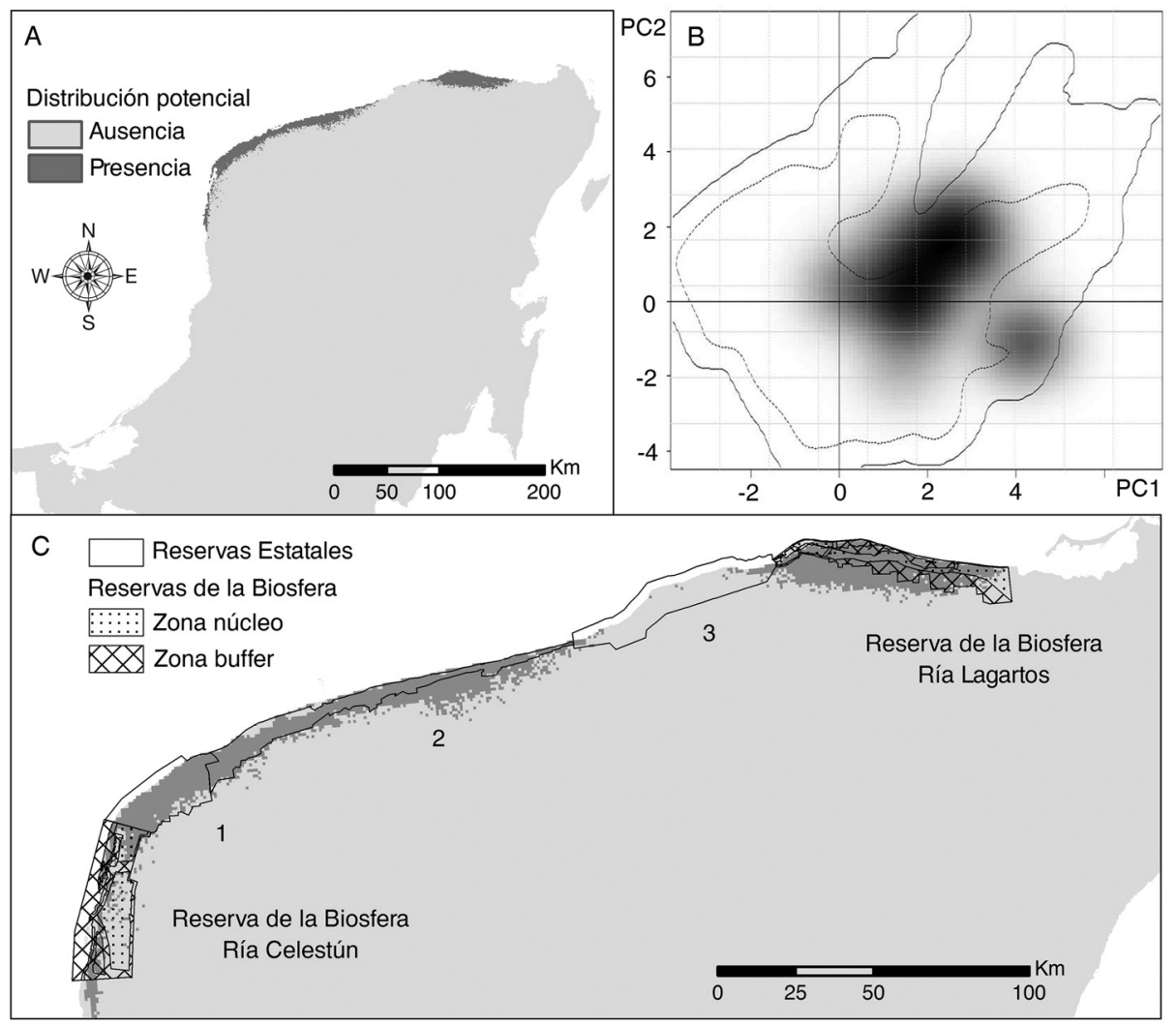

Fig. 1. A. Modelo de distribución potencial de Campylorhynchus yucatanicus en la Península de Yucatán obtenidos en MaxEnt con las variables empleadas; B. modelo de nicho ambiental de la especie graficando los dos primeros componentes del PCA donde la intensidad de sombreado gris muestra la densidad de ocurrencias de la especie en cada celda y las curvas de nivel continuas y discontinuas ilustran el $100 \%$ y $50 \%$ respectivamente, del medio ambiente disponible: y C. Ubicación de las Reservas de la Biosfera presentes en la zona y las Reservas Estatales: (1) El Palmar, (2) Humedales y Manglares de la costa norte de Yucatán, (3) Dzilam.

Fig. 1. A. Potential distribution model of Campylorhynchus yucatanicus in the Yucatán Peninsula, obtained in MaxEnt with the variables used; B. Model of environmental niche of this species, the graph show the plot of the two first components of PCA. The intensity of gray shading shows the density of occurrence of the species in each pixel, and the continuous and discontinuous curves illustrate the environmental available, $100 \%$ and $50 \%$ respectively; and C. Location of Biosphere Reserves present in the area and the State Reserves: (1) El Palmar, (2) Humedales y Manglares de la costa norte de Yucatán, (3) Dzilam.

distribución potencial estuvo dentro de alguna de las tres ANP estatales, que contienen el $66 \%$ $\left(1780 \mathrm{~km}^{2}\right): 2 \%$ en RE Dzilam $\left(48 \mathrm{~km}^{2}\right), 21 \%$ en la RE Ciénagas y manglares de la costa norte de Yucatán $\left(580 \mathrm{~km}^{2}\right)$ y $15 \%$ en la RE El Palmar $\left(414 \mathrm{~km}^{2}\right)$. La menor proporción de área de distribución potencial se encontró en las RB, estando el $20 \%$ en la RBRL $\left(543 \mathrm{~km}^{2}\right)$, y el $7 \%$ en RBRC $\left(282 \mathrm{~km}^{2}\right)$. Además, sólo el $10 \%$ del área potencial se encontró protegida dentro de alguna zona núcleo de las RB (7 \% en RBRL y $3 \%$ en RBRC), lo que corresponde a una pequeña área de $282 \mathrm{~km}^{2}$.

Conectividad del paisaje: Las variables analizadas y el mapa de condiciones favorables (Fig. 3A) permitieron obtener un modelo de conectividad del paisaje para el probable movimiento de individuos entre poblaciones (Fig. 3B). De manera general, el modelo predijo 

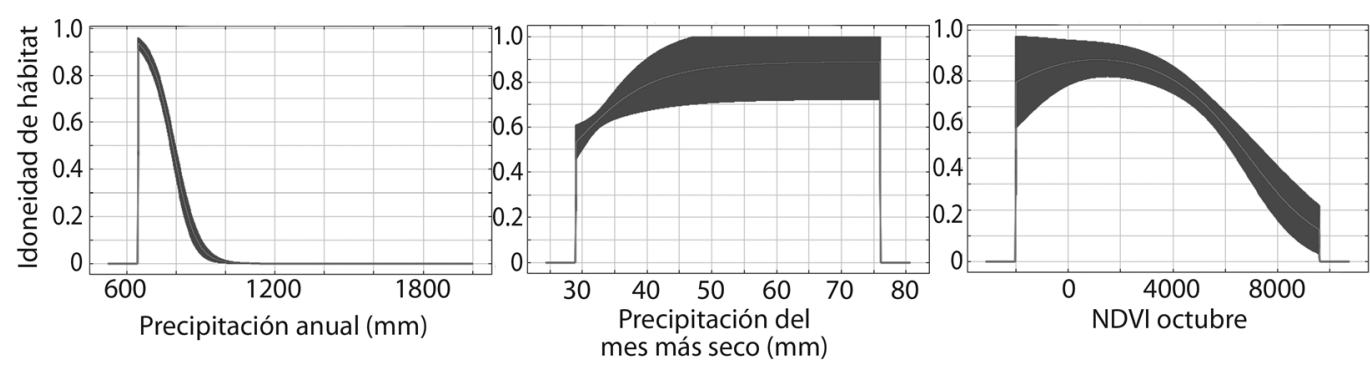

Fig. 2. Curvas de respuesta de las variables ambientales que tuvieron mayor porcentaje de contribución a los modelos de distribución potencial (> 10 \%) de Campylorhynchus yucatanicus en la Península de Yucatán.

Fig. 2. Response curves of environmental variables with the greatest percentage contribution to potential distribution models (>10\%) of Campylorhynchus yucatanicus in the Yucatan Peninsula.

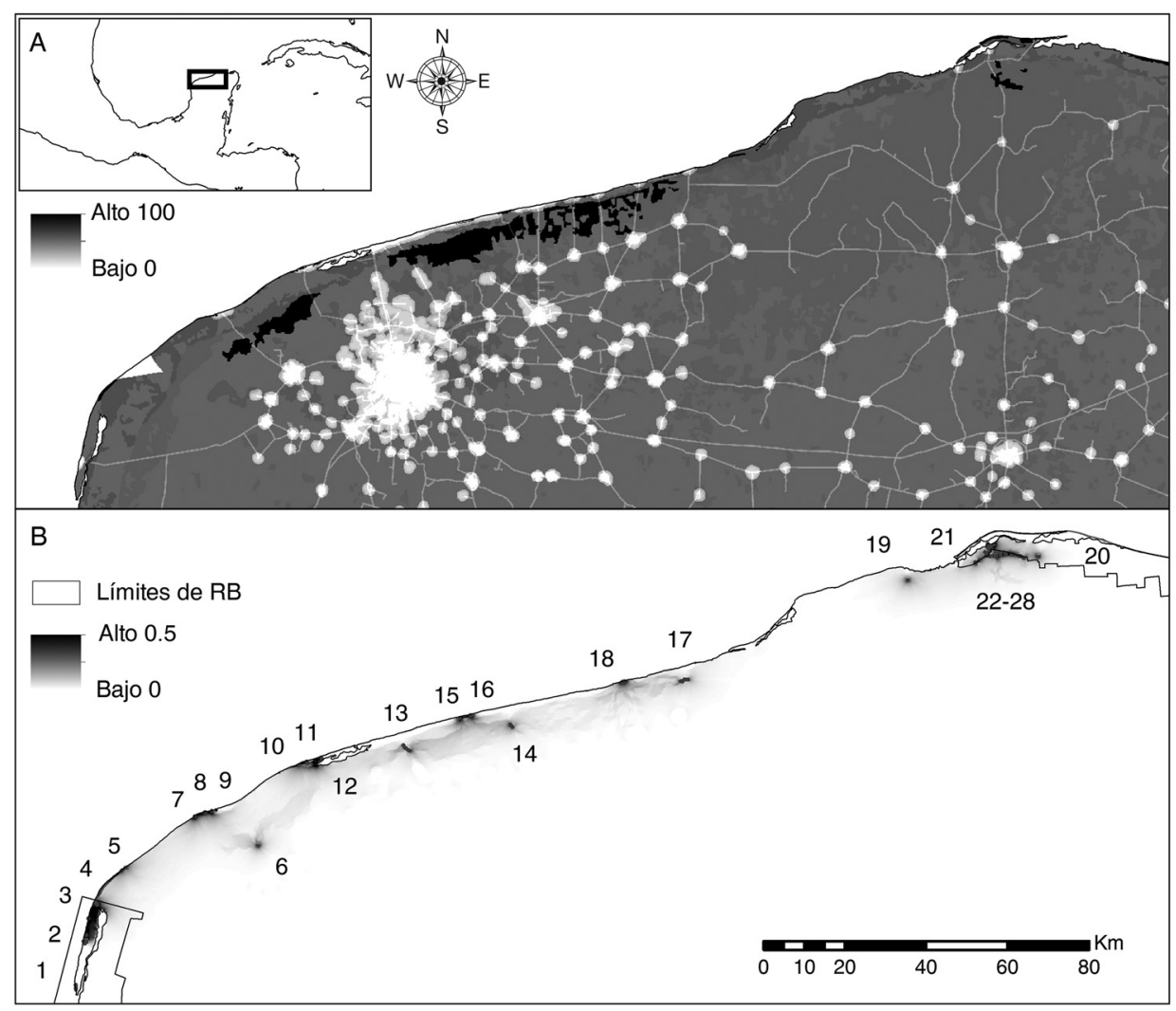

Fig. 3. Mapa de permeabilidad del paisaje para Campylorhynchus yucatanicus teniendo en cuenta los tipos de vegetación y uso de suelo, la distancia a los asentamientos humanos y a las carreteras asfaltadas (A) y modelo de conectividad entre poblaciones de C. yucatanicus representando la conductancia entre nodos focales (B). RB: Reserva de la Biosfera.

Fig. 3. Map of landscape permeability for Campylorhynchus yucatanicus considering vegetation type and land use, distance to human settlements and paved roads (A) and model connectivity among populations of C. yucatanicus representing the conductance between focal nodes (B). 
que puede existir cierta conectividad entre la mayoría de las poblaciones de C. yucatanicus. Sin embargo, hubo grupos de poblaciones que pudieran estar más aisladas del resto. Por ejemplo, la población 1 dentro de la RBRC, en el extremo más occidental de la distribución de la especie, está potencialmente aislada del resto de las poblaciones. Así mismo, el grupo de nodos focales ubicados en la zona oriental de la distribución, del 19 al 28, no parecen tener relación con los demás nodos focales. La conectividad entre poblaciones más alejadas fue relativamente baja y estuvo favorecida, en la mayoría de los casos, por los fragmentos de vegetación secundaria arbustiva y arbórea de selva baja espinosa caducifolia (más alejados de la línea de costa), y no por la vegetación de dunas costeras.

\section{DISCUSIÓN}

La distribución potencial obtenida para la especie en este trabajo incluyó las localidades que reportan anteriormente otros autores (e.g. Paynter, 1955; Zimmerman, 1957; Howell \& Webb, 1995; Vargas-Soriano et al., 2010), ubicándose por la mayor parte del litoral costero desde el extremo norte de Campeche hasta el límite este de la RBRL. Sin embargo, el modelo de distribución potencial que aquí se presentó, difiere en extensión del mapa que reporta CONABIO (Navarro-Sigüenza \& Peterson, 2007), ya que se detectó que existe menor área con condiciones favorables para la especie. En el área comprendida entre el poblado de Dzilam de Bravo hasta los alrededores de la localidad de San Felipe, hubo una baja representación del área de distribución potencial de la especie, que puede estar dada porque en dicha zona el manglar se desarrolla hasta la orilla de la línea de costa. Por esta razón, en esta porción de la línea costera las dunas son escasas o inexistentes y no se encuentra el hábitat adecuado para el desarrollo de la especie. En la ANP de Dzilam, por ejemplo, se pueden encontrar fragmentos con vegetación secundaria de selva baja caducifolia espinosa, pero esta ha sufrido grandes modificaciones por el extensivo uso para la ganadería, la agricultura y la extracción de roca caliza, incluso dentro de las áreas destinadas a la protección (Lamb, Erksine, \& Parotta, 2005). Las modificaciones de la vegetación que ahí se han encontrado (Leirana-Alcocer, Hernández-Betancourt, Salinas-Peba, \& Guerrero-González, 2009), podrían estar influyendo negativamente en la presencia de la especie.

Los registros de C. yucatanicus más alejados de la línea de costa obtenidos por nosotros en trabajo de campo se ubican a $7 \mathrm{~km}$ de la playa, mientras que MacKinnon (2013) refiere que lo más alejado de la costa que ha detectado la especie es a $5 \mathrm{~km}$. En los modelos obtenidos con las líneas de corte según los algoritmos MTP, ETSS y MTSPS, la especie presentó una distribución potencial a más de $15 \mathrm{~km}$ de la zona costera. Esta fue una de las razones por las cuales no se tomaron en cuenta y se optó por tomar como el mejor mapa binario el que se obtuvo con la opción 10P, que si cumplía con este requerimiento de la especie. Atendiendo a este criterio y a la experiencia en campo, los mapas de distribución potencial propuestos por Navarro-Sigüenza y Peterson (2007) también sobrestiman el área de distribución potencial de la especie.

La mayor parte del área de distribución que se obtuvo para la $C$. yucatanicus está dentro del estado de Yucatán. Aun cuando casi toda esta zona costera se encuentra dentro de alguna ANP de tipo federal o estatal, algunos autores recomienda proteger el $100 \%$ del área de distribución de las especies cuando estas son cercanas o menores a $1000 \mathrm{~km}^{2}$ (Rodrigues et al., 2004). Por otro lado, los Planes de Manejo en las ANP no han existido siempre, y los que hay no contemplan en general que el manejo de los recursos se fundamenta en el entendimiento de los procesos funcionales de los ecosistemas (Herrera, Comín, \& Capurro, 2004). En la zona de estudio, las dunas costeras están siendo destruidas a lo largo de $25 \mathrm{~km}$ a ambos lados de la ciudad de Progreso, por el alargamiento de las zonas urbanas de los pueblos costeros, la construcción de carreteras, las áreas de contenedores de carga y la urbanización para el turismo 
semintensivo (Herrera et al., 2004). A lo largo de la costa norte de la Península de Yucatán se tiene conocimiento de construcciones de viviendas en donde ocurría el matorral costero, incluso dentro de las ANP.

Por esta razón, el hábitat de C. yucatanicus está siendo destruido principalmente en esta zona, ya que la demanda de espacios para recreación durante el verano por los habitantes de la ciudad de Mérida y zonas aledañas es cada día mayor. Aunado a esto, los procesos que han impactado los ecosistemas costeros se han ido amplificando, con lo que ha sido evidente la pérdida de hábitats críticos y se ha incrementado la contaminación orgánica (Euán \& Scout, 2002, Herrera et al., 2002). En muchas de estas áreas no existen sistemas de alcantarillado y de tratamiento de aguas residuales, con lo que la salud de los ecosistemas se ve seriamente amenazada (Bricker, Clement, Pirhalla, Orlando, \& Farrow, 1999). Además, se debe tener en cuenta que la calidad del agua en las lagunas costeras y en la zona marina adyacente se ha reducido a consecuencia de las descargas de aguas residuales urbanas e industriales (Herrera et al., 2002). La vegetación secundaria de selva baja espinosa caducifolia, y la secundaria de manglar arbustivo, donde también pueden encontrarse poblaciones de C. yucatanicus, también están siendo modificadas y fragmentadas. Una vez eliminada la vegetación primaria, los principales usos que se les da son la agricultura, la ganadería, la explotación de madera, la producción de carbón vegetal, entre otros. Es necesario reforzar la vigilancia y protección dentro de las ANP, así como los mecanismos de regulación legales dirigidos a la protección de estas áreas, el hábitat y la flora y fauna asociados a ellas.

Las RB y las RE presentes en la zona de estudio cubren la mayoría de la costa norte de la Península de Yucatán, por lo cual se puede decir que la costa se encuentra en alguna categoría de protección. Sin embargo, al menos en estas RE no consta ninguna estrategia de conservación eficiente que permita preservar una adecuada conectividad entre poblaciones de C. yucatanicus. Los modelos de conectividad que obtuvimos y los valores de resistencia entre los nodos muestran la importancia de los fragmentos de hábitat para lograr una conectividad entre las poblaciones de esta especie en peligro. Se hace urgente establecer medidas de conservación de su hábitat fuera de las RB, especialmente aquellos con vegetación de duna costera, que al parecer son los más amenazados por falta de conectividad. Las poblaciones más aisladas son las de los extremos de la distribución, y requieren de atención para la conservación. Los individuos de RBRC al parecer están aislados del resto de las poblaciones por la presencia del pueblo de Celestún hacia el este, y por los cuerpos de agua, los cuales podría constituir barreras, teniendo en cuenta la conducta de la especie. Los asentamientos humanos como Celestún, Chuburná Puerto, entre otros, podrían constituir barreras relativamente recientes en la historia evolutiva de la especie, pero no se conoce qué efecto tienen en la preservación de la especie. Las poblaciones potencialmente aisladas podrían ser objetivos en los planes de manejo para la conservación.

Patrones similares de aislamiento por fragmentación de hábitat se han encontrado en escalas geográficas pequeñas para una especie del mismo género. Barr et al. (2015) encontraron que las diferencias genéticas entre poblaciones de $C$. brunneicapillus relativamente cercanas podían estar relacionadas con fenómenos de urbanización, incendios, y fragmentación del hábitat. La tasa de dispersión de C. brunneicapillus estimada por estos investigadores fue relativamente baja y constituye un factor a tener en cuenta en los modelos de conectividad de estas especies.

La inferencia sobre el impacto de la fragmentación del hábitat en la dispersión y el flujo de individuos y genes es un tema clave en biología de conservación. Recientemente, se ha cuestionado la capacidad de los enfoques establecidos para identificar con fiabilidad los efectos de la estructura del paisaje (por ejemplo, la composición de la cubierta terrestre, la configuración y extensión de la vegetación remanente) sobre la movilidad de los organismos (Manel, Gaggiotti, \& Waples, 2005). 
Por ende, los métodos para predecir y probar esos efectos son cada vez más explícitos, integran diferentes disciplinas (genética poblacional, ecología, estadística espacial entre otros) y utilizan volúmenes de información mayores y más complejos para obtener mejores modelos de conectividad (Garrido-Garduño \& VázquezDomínguez, 2013). Sin embargo, estos estudios integradores demandan mucho tiempo, gran esfuerzo de muestreo y recursos antes de obtener resultados aplicables a la conservación. Además, algunos investigadores han probado la utilidad del conocimiento empírico acerca de la historia natural en estos modelos (Amos et al., 2012). El modelo de conectividad encontrado por nosotros es solo un primer intento de aproximarnos a la realidad. De cualquier manera, es fundamental realizar estudios de genética poblacional y de captura-marcaje-recaptura, para comprender de forma certera cómo se comporta la movilidad y dispersión de los individuos de C. yucatanicus y la conectividad entre los fragmentos de vegetación ocupada.

La información obtenida en este trabajo es útil para reevaluar la categoría de amenaza de la especie a nivel internacional. Consideramos que existen evidencias de que la especie se encuentra en categoría de en peligro de la UICN teniendo en cuenta el criterio B correspondiente a la extensión de la distribución estimada, que es menor de $5000 \mathrm{~km}^{2}$ (UICN, 2012). El hábitat de la especie está fragmentado y las amenazas son las mismas en casi la totalidad de la distribución (Criterio B-1-a). Aunque no se tienen datos precisos, es posible que haya ocurrido una disminución de las áreas de ocupación de la especie (Criterio B-1-c), ya que se tienen registros históricos de presencia en lugares donde ya no podemos encontrarla, por ejemplo, en las cercanías de Isla Arena, $\mathrm{y}$ algunos puntos que ahora quedan dentro de asentamientos humanos.

Los modelos obtenidos deben tratarse con cuidado debido al grado de incertidumbre que pueden tener intrínsecos en el método (Pearson et al., 2006). Sin embargo, con los resultados obtenidos se cuenta con la información necesaria para definir prioridades respecto a la conservación de la especie dentro del área de distribución predicha. Este estudio indicó que C. yucatanicus se encuentra en un estado de conservación más delicado del que hasta ahora se había descrito al menos en la UICN. Deben tenerse en cuenta para el manejo de la especie aquellas áreas que actualmente perfilan como las de mayor importancia y las poblaciones más sensibles por su grado de aislamiento.

Métodos similares a los utilizados en este estudio han sido útiles para establecer áreas de prioridad para la conservación de otras especies de aves endémicas y amenazadas en las zonas tropicales (Marini, Barbet-Massin, Lopes, \& Jiguet, 2010; de Carvalho et al., 2017). El presente estudio servirá también de referencia en el futuro para evaluaciones similares de especies con distribución restringida. Adicionalmente, nuestros resultados marcan pautas para próximas evaluaciones de riesgos ante cambio climático, por su cercanía a las costas bajas de la Península de Yucatán, y podrán ser punto de partida para predecir invasiones de especies e identificar áreas potenciales para reintroducción (Wilson, Westphal, Possingham, \& Elith., 2005; Broennimann \& Guisan, 2008; Marini, Barbet-Massin, Lopes, \& Jiguet, 2009).

\section{AGRADECIMIENTOS}

Agradezco a los que ayudaron durante el trabajo de campo, Guillermo E. Castillo Vela y José A. España España, y al personal de las ANP que nos recibieron. A los especialistas y observadores de aves que nos proporcionaron información valiosa, Barbara McKinnon, Alexander Dzib. A ECOSUR y Conacyt por el apoyo a través del programa de becas de doctorado. A The Rufford Foundation y Cornell Lab of Ornithology por el apoyo económico. A Idea Wild y Birder Exchange por los equipos donados.

\section{RESUMEN}

La distribución geográfica y la calidad del hábitat son criterios fundamentales para evaluar el grado de riesgo de extinción y amenaza de las especies. Campylorhynchus 
yucatanicus es un ave endémica de la costa norte de la Península de Yucatán, México con distribución restringida a una estrecha franja de hábitat, entre los estados de Campeche y Yucatán. En la actualidad, la costa yucateca ha perdido más de la mitad de la vegetación de duna costera y otros hábitats se han modificado principalmente por falta de un plan de urbanización y mal aprovechamiento de los recursos naturales. Estos factores amenazan a C. yucatanicus, que está catalogada como una especie casi amenazada por la UICN y como una especie en peligro de extinción por la legislación mexicana, NOM-059-2010. En este estudio se modeló su distribución potencial utilizando 64 registros de presencia de variadas fuentes (1960 y 2009), un conjunto de variables climáticas y una capa de Índice de vegetación de diferencia normalizada (NDVI). Para evaluar el grado de conectividad del paisaje se empleó un mapa de tipos de vegetación y uso de suelo, distancia a los poblados y a las carreteras asfaltadas. El modelo de distribución potencial arrojó una distribución potencial de $2711 \mathrm{~km}^{2}$ aproximadamente, $2 \%$ del área total de la Península de Yucatán. De esta área, solo el 27 \% está protegido con categoría de Reserva de la Biósfera y sólo el 10 \% corresponde a áreas núcleo de conservación, con restricciones de uso de suelo y protección relativamente efectiva. Las poblaciones de la región de Ría Lagartos y del oeste de Celestún, parecen ser las más aisladas, teniendo en cuenta el modelo de conectividad del paisaje. La permeabilidad del paisaje entre fragmentos de vegetación de duna cerca de la costa es baja, principalmente por la distribución de las zonas urbanas. Los resultados pueden ser usados para establecer estrategias de manejo y sugieren que la especie se encuentra en un estado de conservación más delicado del que hasta ahora se había descrito por la UICN. Consideramos que C. yucatanicus debe tener categoría en peligro de la UICN según su distribución y la conectividad de paisaje actual.

Palabras clave: endémica, amenazada, matraca yucateca, distribución potencial, conectividad.

\section{REFERENCIAS}

Alaniz, A. J., Galleguillos, M., \& Perez-Quezada, J. F. (2016). Assessment of quality of input data used to classify ecosystems according to the IUCN Red List methodology: The case of the central Chile hotspot. Biological Conservation, 204, 378-385. doi 10.1016/j.biocon.2016.10.038

Amos, J. N., Bennett, A. F., Mac Nally, R., Newell, G., Pavlova, A., Radford, J. Q., Thomson, J. R., White, M., \& Sunnucks P. (2012). Predicting LandscapeGenetic Consequences of Habitat Loss, Fragmentation and Mobility for Multiple Species of Woodland Birds. PLoS ONE, 7(2), e30888. doi:10.1371/journal. pone. 0030888

Barker, F. K. (2007). Avifaunal interchange across the Panamanian Isthmus: Insights from Campylorhynchus wrens. Biological Journal of the Linnean Society, 90, 687-702.

Barr, K. R., Kus, B. E., Preston, K. L., Howell, S., Perkins, E., \& Vandergast, A. G. (2015). Habitat fragmentation in coastal southern California disrupts genetic connectivity in the cactus wren (Campylorhynchus brunneicapillus). Molecular Ecology, 24, 2349-2363.

Barve, N. (2008). Tool for partial-ROC (Biodiversity Institute, Lawrence, KS), ver 1.0.

Bellard, C., Bertelsmeier, C., Leadley, P., Thuiller, W. \& Courchamp, F. (2012). Impacts of climate change on the future of biodiversity. Ecology Letters, 15, 365377. doi:10.1111/j.1461-0248.2011.01736.x

Berlanga, H., Gómez, H., Vargas, V. M., Rodríguez, V., Sánchez, L. A., Ortega, R., \& Calderón, R. (2015). Aves de México. Lista actualizada de especies y nombres comunes. México D. F, México: Comisión Nacional para el Conocimiento y Uso de la Biodiversidad (CONABIO).

Bezaury-Creel, J., \& Gutiérrez-Carbonell, D. (2009). Áreas naturales protegidas y desarrollo social en México. In R. Dirzo, R. González, I. J. March (Eds.), Capital natural de México, Vol. II: Estado de conservación y tendencias de cambio (pp. 385-431). México D. F., México: CONABIO.

Bricker, S. B., Clement, C. G., Pirhalla, D. E., Orlando, S. P., \& Farrow, D. R. G. (1999). National estuarine eutrophication assessment: Effects of nutrient enrichment in the Nation's estuaries (NOAA, National Ocean Service, Special Projects Office, and the National Center for Coastal Ocean Science). Maryland, USA: Silver Spring.

Broennimann, O., Fitzpatrick, M. C., Pearman, P. B., Petitpierre, B., Pellissier, L., Yoccoz, N. G., Thuiller, W., Fortin, M. J., Randin, C., Zimmermann, N. E., Graham, C. H., \& Guisan, A. (2012). Measuring ecological niche overlap from occurrence and spatial environmental data. Global Ecology and Biogeography, 21, 481-497.

Broennimann, O., \& Guisan, A. (2008). Predicting current and future biological invasions: both native and invaded ranges matter. Biology Letters, 4, 585-589.

Ceballos, G., Gómez Silva, G., \& del Coro Arizmendi M. (2002). Áreas prioritarias para la conservación de las aves en México. CONABIO. Biodiversitas, 41, 1-7.

Cuervo, A. P., Téllez, O., Gómez, M., Venegas, C., Manjarrez, J., \& Martínez, E. (2013). An update of high-resolution monthly climate surfaces for Mexico. International Journal of Climatology, 34, 2427-2437.

CONANP. (2000). Programa de Manejo de la Reserva de la Biosfera Ría Celestún. Comisión Nacional de Áreas Naturales Protegidas, Estado de Yucatán, México. 191 p. 
Correa Ayram, C. A., Mendoza, M. E., Etter, A., \& Pérez Salicrup, D. R. (2016). Habitat connectivity in biodiversity conservation: A review of recent studies and applications. Progress in Physical Geography, 40(1), 7-37.

De Carvalho, D. L., Sousa-Neves, T., Cerqueira, P. V., Gonsioroski, G., Silva, S. M., Silva, D. P., Paiva Silva, D., \& Dantas Santos, M. P. (2017). Delimiting priority areas for the conservation of endemic and threatened Neotropical birds using a niche-based gap analysis. PLoS ONE, 12(2), e0171838. doi:10.1371/journal. pone. 0171838

Del Hoyo, J., Elliot, A., \& Christie, D. (2005). Handbook of the birds of the world (Volume 10: Cuckoo-shrikes to trushes). Barcelona, España: Lynx Edicions.

Durán, R., Torres, W. M., \& Espejel, I. (2010). Vegetación de dunas costeras. In R. Durán, M. Méndez, \& J. Arellano (Eds.), Diversidad biológica y desarrollo humano en Yucatán (pp. 136-137). Yucatán, México: CICY, SEDUMA PNUD, CONABIO.

Dyer, E. E., Franks, V., Cassey, P., Collen, B., Cope, R. C., Jones, K. E., Sekercioglu, C. H., \& Blackburn, T. M. (2016). A global analysis of the determinants of alien geographical range size in birds. Global Ecology and Biogeography, 25(11), 1346-1355. DOI: 10.1111/ geb. 12496

Elith, J., \& Graham, C. H. (2006). Novel methods improve prediction of species' distributions from occurrence data. Ecography, 29, 129-151.

Espejel, I. (1984). La vegetación de las dunas costeras de la Península de Yucatán. I. Análisis florístico del estado de Yucatán. Biótica, 9(2), 183-210.

Espejel, I. (1987). A phytogeographical analysis of coastal vegetation in the Yucatan Peninsula. Journal of Biogeography, 14, 499-519.

Euán, J. \& Scout, W. G. (2002). Promoting integrated coastal management in the Yucatan Peninsula, México. Journal of Policy Studies, 12, 1-16.

Flores, J. S., \& Espejel, I. (1994). Tipos de Vegetación de la Península de Yucatán. Etnoflora Yucatanense. Fascículo 3. Yucatán, México: Universidad Autónoma de Yucatán.

Garrido-Garduño, T., \& Vázquez-Domínguez, E. (2013). Métodos de análisis genéticos, espaciales y de conectividad en genética del paisaje. Revista Mexicana de Biodiversidad, 84, 1031-1054.

García, E. (2004). Modificaciones al sistema de clasificación climática de Köppen. México DF: Serie libros. Instituto de Geografía-UNAM.

Guisan, A., Broennimann, O., Engler, R., Vust, M., Yoccoz, N. G., Lehmann, A., \& Zimmermann, N. E. (2006). Using Niche-Based Models to Improve the
Sampling of Rare Species. Conservation Biology, 20(2), 501-511.

Herrera, J. A, Medina, I., Arandal, N., Zaldivar, J. A., Ramírez, J., \& Trejo, J. (2002). Trophic status in coastal waters of the Yucatán Península (SE, México) using water quality indicators. In C. A. Brebbia (Ed.), Coastal Environment. Environmental Problems in Coastal Regions IV (pp. 351-359). Southampton, England: Wessex Institute of Technology.

Herrera, J. A., Comín, F. A., \& Capurro, L. (2004). Los usos y abusos de la zona costera en la Península de Yucatán. In E. Rivera, G. J. Villalobos, I. Azuz \& F. Rosado (Eds.), El Manejo Costero en México (pp. 387-396). Quintana Roo, México: Universidad Autónoma de Campeche, SEMARNAT, CETYSUniversidad, Universidad de Quintana Roo.

Howell, S., \& Webb, S. (1995). A Field Guide to the Birds of México and Northern Central America. Nueva York, USA: Oxford University Press.

INEGI. (2013). Conjunto Nacional de Uso de Suelo y Vegetación a escala 1:250000. Serie V, DGG-INEGI. México: Instituto nacional de Estadística y Geografía (INEGI). Recuperado de http://www.inegi.org.mx/

INEGI. (2014). Guía para la interpretación de cartografia: uso del suelo y vegetación: escala 1:250, 000: Serie $V$. México: Instituto nacional de Estadística y Geografía (INEGI). Recuperado en http://www.inegi. org.mx/geo/

IUCN. (2015). The IUCN red list of threatened species. Version 2015-4. Recuperado en http://www.iucnredlist.org

Keith, D. A. (2015). Assessing and managing risks to ecosystem biodiversity. Austral Ecology, 40, 337-346.

Koen, E. L., Garroway, C. J., Wilson, P. J., \& Bowman, J. (2010). The effect of map boundary on estimates of landscape resistance to animal movement. PLoS ONE, 5(7), e11785. doi:10.1371/journal. pone. 0011785

Kozak, K. H., Graham, C. H., \& Wiens, J. J. (2008). Integrating GIS-based environmental data into evolutionary biology. Trends in Ecology \& Evolution, 23, 141-148.

Lamb, D., Erksine, P. D., \& Parotta, J. A. (2005). Restoration of tropical forest landscapes. Science, $310,1628-1632$.

Leirana-Alcocer, J. L., Hernández-Betancourt, S., SalinasPeba, L., \& Guerrero-González, L. (2009). Cambios en la estructura y composición de la vegetación relacionados con los años de abandono de tierras agropecuarias en la selva baja caducifolia espinosa de la Reserva de Dzilam, Yucatán. Polibotánica, 27, 51-68. 
Liu, C., Berry, P. M., Dawson, T. P., \& Pearson, R. G. (2005). Selecting thresholds of occurrence in the prediction of species distributions. Ecography, 28, 385-393.

MacKinnon, B. (2013). Sal a pajarear Yucatán. Guía de aves. México D. F, México: La Vaca Independiente S.A. de C.V.

Marini, M. A., Barbet-Massin, M., Lopes, L. E., \& Jiguet, F. (2009). Predicted climate-driven bird distribution changes and forecasted conservation conflicts in a neotropical savanna. Conservation Biology, $23,1558-1567$.

Marini, M. A., Barbet-Massin, M., Lopes, L. E., \& Jiguet, F. (2010). Predicting the occurrence of rare Brazilian birds with species distribution models. Journal Ornithology, 151, 857-866.

Maass, M., Jardel, E., Martínez-Yrízar, A., Calderón, L., Herrera, J., Castillo, A., Euán-Ávila, J., \& Equihua, M. (2010). Las áreas naturales protegidas y la investigación ecológica de largo plazo en México. Ecosistemas, 19(2), 69-83.

McRae, B. H., \& Shah, V. B. (2009). Circuitscape user's guide. California, Estados Unidos: The Univeristy of California, Santa Barbara. Recuperado en http:// www.circuitscape.org

McRae, B. H., Dickson, B. G., Keitt, T. H., \& Shah, V. B. (2008). Using circuit theory to model connectivity in ecology, evolution, and conservation. Ecology, $89(10), 2712-2724$

Modest, R. B., \& Hassan, S. N. (2016). Species Composition of Tropical Understory Birds in Threatened East African Coastal Forests Based on Capture Data. International Journal of Zoology, V2016, 9. doi. org/10.1155/2016/1390364

Naranjo, E. J., Dirzo, R., López, J. C., Rendón-von, J., Reuter, A., \& Sosa-Nishizaki, Ó. (2009). Impacto de los factores antropogénicos de afectación directa a las poblaciones silvestres de flora y fauna, en Capital natural de México, Vol. II. In Estado de conservación y tendencias de cambio (pp. 247-276). México: Comisión Nacional para el Conocimiento y Uso de la Biodiversidad (CONABIO).

Navarro-Sigüenza, A. G., \& Peterson, A. T. (2007). Campylorhynchus yucatanicus (matraca yucateca) residencia permanente. Distribución potencial. In: Mapas de las aves de México basados en $W W W$ (Informe final SNIB-CONABIO proyecto No. CE015). México D. F.: Museo de Zoología, Facultad de Ciencias, UNAM \& University of Kansas, Museum of Natural History, Comisión Nacional para el Conocimiento y Uso de la Biodiversidad (CONABIO).

Paynter, R. A. (1955). The Ornithogeography of the Yucatan Peninsula. New Haven, Connecticut: Museum of Comparative Zoology Harvard University.
Pearson, R. G., Thuiller, W., Araújo, M. B., MartinezMeyer, E., Brotons, L., McClean, C., Miles, L., Segurado, P., Dawson, T. P., \& Lees, D. C. (2006). Model-based uncertainty in species range prediction. Journal of Biogeography, 33, 1704-1711.

Peterson, A. T, Papes, M., \& Soberon, J. (2008). Rethinking receiver operating characteristic analysis applications in ecological niche modeling. Ecological Modelling, 213, 63-72.

Phillips, S. J., Anderson, R. P., \& Schapire, R. E. (2006). Maximum entropy modeling of species geographic distributions. Ecological Modelling, 190, 231-259.

Rabenold, K. N. (1990). Campylorhynchus wrens: The ecology of delayed dispersal and cooperation in the Venezuelan savanna. In P. B. Stacey, \& W. D. Koenig (Eds.), Cooperative breeding in birds: long-term studies of ecology and behavior (pp. 157-196). United Kingdom: Cambridge University Press.

R Development Core Team. 2014. A language and environment for statistical computing. $\mathrm{R}$ Foundation for Statistical Computing, Vienna, Austria. ISBN 3-900051-07-0, URL http://www.R-project.org.

Rödder, D., Nekum, S., Cord, A. F., \& Engler, J. O. (2016). Coupling satellite data with species distribution and connectivity models as a tool for environmental management and planning in matrix-sensitive species. Environmental Management, 58(1), 130-143. doi 10.1007/s00267-016-0698-y

Rodrigues, A. S. L., Akçakaya, H. R., Andelman, S. J., Bakarr, M. I., Boitani, L., Brooks, T. M., Chanson, J. S., Fishpool, L. D. C., da Fonseca, G. A. B., Gaston, K. J., Hoffmann, M., Marquet, P. A., Pilgrim, J. D., Pressey, R. L., Schipper, J., Sechrest, W., Stuart, S. N., Underhill, L. G., Waller, R. W., Watts, M. E. J., \& Yan, X. (2004). Global Gap Analysis: Priority Regions for Expanding the Global Protected-Area Network. BioScience, 54(12), 1092-1100.

SEMARNAT (Secretaría de Medio Ambiente y Recursos Naturales). (2010). Norma Oficial Mexicana NOM059-SEMARNAT-2010. Protección Ambiental. Especies nativas de México de flora y fauna silvestres. Categorías de riesgo y especificaciones para su inclusión, exclusión o cambio. Lista de especies en riesgo. Diario Oficial de la Federación. 30 de diciembre de 2010, Segunda Sección. México, DF: SEMARNAT.

Shimazaki, A., Yamaura, Y., Senzaki, M., Yabuhara, Y., Akasaka, T., \& Nakamura, F. (2016). Urban permeability for birds: An approach combining mobbing-call experiments and circuit theory. Urban Forestry \& Urban Greening, 19(1), 167-175.

Silverman, B. W. (1986). Density estimation for statistics and data analysis. London, England: Chapman \& Hall. 
Stotz, D. F., Fitzpatrick, J. W., Parker III, T. A., \& Moskovits, D. K. (1996). Neotropical birds: ecology and conservation. Illinois, USA: The University of Chicago Press, Chicago.

Taylor, P. D., Fahrig, L., Henein, K., \& Merriam G. (1993). Connectivity is a vital element of landscape structure. Oikos, 68, 571-573.

Tischendorf, L., \& L. Fahrig. (2000). How should we measure landscape connectivity? Landscape Ecology, 15(7), 633-641.

UICN. (2012). Categorías y Criterios de la Lista Roja de la UICN: Versión 3.1. (2nd edition). Gland, Suiza y Cambridge, Reino Unido: UICN.

Vargas-Soriano, J., Salgado-Ortíz, J., \& Escalona-Segura, G. (2010). Breeding phenology and nesting success of the Yucatan Wren in the Yucatan Peninsula, Mexico. The Wilson Journal of Ornithology, 122(3), 439-446.
Vázquez-Miranda, H. A., Navarro-Sigüenza, G., \& Omland, K. E. (2009). Omland. Phylogeography of the Rufous-Naped wren (Campylorhynchus rufinucha): speciation and hybridization in Mesoamerica. The Auk, 126(4), 765-778.

Warren, D. L., Glor, R. E., \& Turelli, M. (2008). Environmental niche equivalency versus conservatism: quantitative approaches to niche evolution. Evolution, $62,2868-2883$.

Warren, D. L., Glor, R. E., \& Turelli, M. (2010). ENMTools: a toolbox for comparative studies of environmental niche models. Ecography, 33, 607-611.

Wilson, K. A., Westphal, M. I., Possingham, H. P., \& Elith, J. (2005). Sensitivity of conservation planning to different approaches to using predicted species distribution data. Biology Conservations, 122, 99-112.

Zimmerman, D. A. (1957). Some remarks on the behavior of the Yucatan Cactus Wren. The Condor, 59, 53-58. 